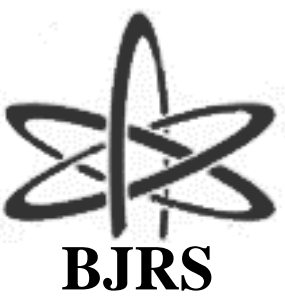

\author{
BRAZILIAN JOURNAL \\ $\mathrm{OF}$ \\ RADIATION SCIENCES \\ 05-03-A (2017) 01-10
}

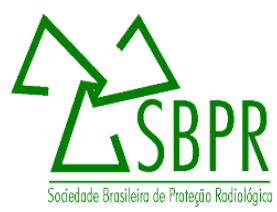

\title{
Análise da variação da atividade de uma amostra de 99m Tc após diluição com soro fisiológico
}

\author{
L. T. Kuahara; E. L. Corrêa; M. P. A. Potiens \\ Gerência de Metrologia das Radiações, Laboratório de Calibração de Instrumentos, \\ Instituto de Pesquisas Energéticas e Nucleares, CEP: 05508-000, \\ São Paulo-SP, Brasil \\ liliankuahara@usp.br
}

\begin{abstract}
RESUMO
O ativímetro é um equipamento essencial em um serviço de medicina nuclear. Garantir seu bom funcionamento e conhecer os fatores que podem influenciar na sua leitura é fundamental para que a atividade administrada ao paciente esteja correta. Vários fatores podem causar alterações na resposta do ativímetro, como o tipo de recipiente, geometria e volume do radioisótopo. $O$ objetivo deste trabalho foi analisar as variações nas medições de 0,5 $\mathrm{mL}$ e $1,0 \mathrm{~mL}$ de ${ }^{99 \mathrm{~m}} \mathrm{Tc}$ puro e diluído em até $2,5 \mathrm{~mL}$ de soro fisiológico, em recipientes utilizados em medicina nuclear. Foram encontradas variações de até $4 \%$ nos valores medidos.
\end{abstract}

Palavras-chave: Ativímetro- medicina nuclear- controle de qualidade

\begin{abstract}
The activimeter is an essential equipment in a nuclear medicine service. Ensuring its proper functioning and knowing the factors that can influence in its reading is fundamental so that the activity administered to the patient is correct. Several factors can cause changes in the response of the activimeter, such as types of containers, geometry, and radioisotope volume. The objective of this work was to analyze the variations in the measurements of $0.5 \mathrm{~mL}$ and $1.0 \mathrm{~mL}$ of ${ }^{99 \mathrm{~m}} \mathrm{Tc}$ pure and diluted in up to $2.5 \mathrm{~mL}$ of solution saline in containers used in nuclear medicine. Variations up to $4 \%$ were found in the measured values.
\end{abstract}

Keywords: activimeter- nuclear medicine-quality control 


\section{INTRODUÇÃO}

A administração de radionuclídeos em pacientes para fins de diagnósticos e terapia tornou-se rotina nos hospitais. A atividade destes radionuclídeos deve ser conhecida com exatidão, a fim de se evitar possíveis danos ao paciente e também otimizar o resultado do exame ou tratamento, mantendo sempre o objetivo de minimizar a dose de acordo com o princípio ALARA ${ }^{(1)}$.

No Brasil os serviços de medicina nuclear possuem um gerador de ${ }^{99 \mathrm{~m}} \mathrm{Tc}$, que é comercializado pelo centro de radiofármacia do Instituto de Pesquisa Energéticas Nucleares (IPEN). Esse gerador vem juntamente com os frascos do IPEN que são semelhantes ao frasco P6, utilizados na eluição do material. A primeira medição da atividade do radiofármaco é realizada neste frasco, quando o material é eluído do gerador e depois é fracionado em uma seringa com uma atividade especifica para a marcação do kit liofilizado que será utilizado.

Estudos anteriores com os frascos P6 e 10R mostraram a necessidade de aplicar fatores de correção toda vez que ocorra uma simples transferência do radiofármaco para outro recipiente, pois este procedimento de transferência ocasiona uma diferença na leitura da atividade da amostra ${ }^{(2)}$.

Além disso, outros fatores podem gerar erros nos resultados apresentados pelo medidor de atividade tais como a posição da fonte dentro da câmara do poço, os tipos de recipientes, geometria e volume dos frascos que contêm esses radiofármacos estudos foram realizados com diversos tipos de recipientes ${ }^{(3)}$.

As medições da atividade de radiofármacos dentro de seringas são uma parte importante do procedimento de preparo do material a ser injetado no paciente. A atividade a ser administrada é geralmente medida nesses recipientes, e não no frasco de vidro do IPEN, que é o enviado pelo fornecedor do radiofármaco. Dessa forma não se pode garantir que a atividade medida nas diferentes etapas do processo de preparo considere todas as variáveis. Para corrigir possíveis erros é preciso utilizar fatores de correção relativos à variação do volume e aplica- los juntamente com os outros fatores citados acima nas medições em diferentes geometrias, com o objetivo de garantir que a medição da atividade seja a mais confiável possível ${ }^{(1)}$. 
A Agência Internacional de Energia Atômica ${ }^{(4)}$ e a Farmacopéia Européia ${ }^{(5)}$ recomendam um desvio máximo $\pm 5 \%$ na exatidão das medições de atividade. Atualmente, no Brasil, a norma CNENNN 3.05 da Comissão Nacional de Energia Nuclear (CNEN) exige um desvio percentual máximo de $\pm 10 \%{ }^{(6)}$.

A norma da CNEN-NN 3.05 também estabelece quais radionuclídeos devem ser utilizados, a periodicidade dos testes e os limites que os ativímetros podem apresentar. No entanto, não existem recomendações claras quanto aos procedimentos para a realização destes testes.

O objetivo deste trabalho é estudar as variações encontradas nas medições da atividade do ${ }^{99 \mathrm{~m}} \mathrm{Tc}$ para dois volumes diferentes de amostra diluídos em soro fisiológico, a fim de analisar de que maneira a distribuição de uma mesma atividade de um determinado radionuclídeo, em diferentes volumes, influencia no resultado apresentado pelo ativímetro, no qual foram determinados os fatores de correção e a incerteza expandida aplicada nas medições que devem ser levados em conta ao se realizar este procedimento de medição .

\section{MATERIAIS E MÉTODOS}

Para a realização deste trabalho foi utilizado o ativímetro Capintec, modelo NPL-CRC, padrão secundário do Laboratório de Calibração de Instrumentos (LCI) do IPEN, rastreável ao National Physical Laboratory (NPL), Inglaterra (figura 1). Por se tratar de um equipamento de referência este ativímetro passa constantemente por testes de controle de qualidade e procedimentos de intercomparação. Todos os resultados obtidos foram satisfatórios, estando dentro dos limites de aceitação, recomendado pela norma CNEN-NN $3.05^{(7-8)}$. 
Figura 1. Ativímetro de referência LCI modelo Capintec NPL-CRC ${ }^{\circledR}$

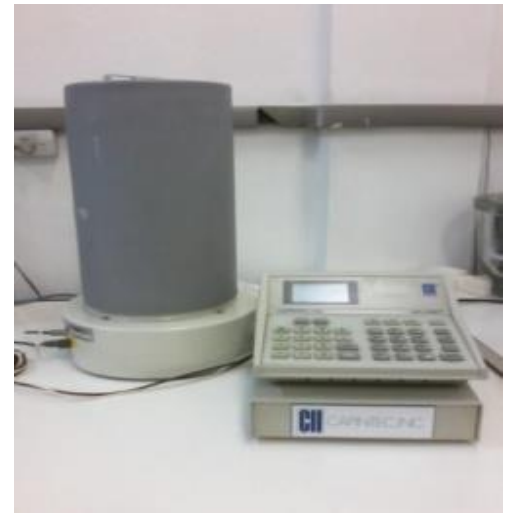

Os testes foram realizados em quatro diferentes recipientes, em dois frascos de vidro sendo o $10 \mathrm{R}$ e o IPEN e em seringas de 3,0 mL e 5,0 mL (figuras 2 e 3). Os testes foram divididos em dois experimentos, um partindo de uma amostra fracionada de $0,5 \mathrm{~mL}$ e outra de $1,0 \mathrm{~mL}$ de ${ }^{99 \mathrm{~m}} \mathrm{Tc}$. A partir daí o volume de cada uma das amostras foi variado adicionando-se $0,5 \mathrm{~mL}$ de soro até completar 3,0 $\mathrm{mL}$ da amostra diluída para cada recipiente.

Figura 2. Frascos de vidro utilizados para medir a amostra de ${ }^{99 m}$ Tc: frasco IPEN e o $10 \mathrm{R}$

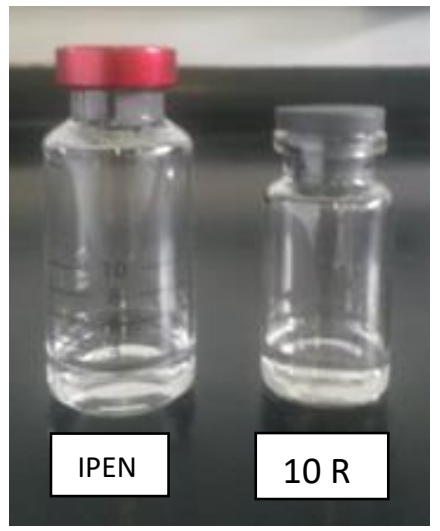


Figura 3. Seringas usadas para medir a amostra de ${ }^{99 \mathrm{~m}} \mathrm{Tc}$ com dois volumes diferentes: $3 \mathrm{e} 5 \mathrm{~mL}$

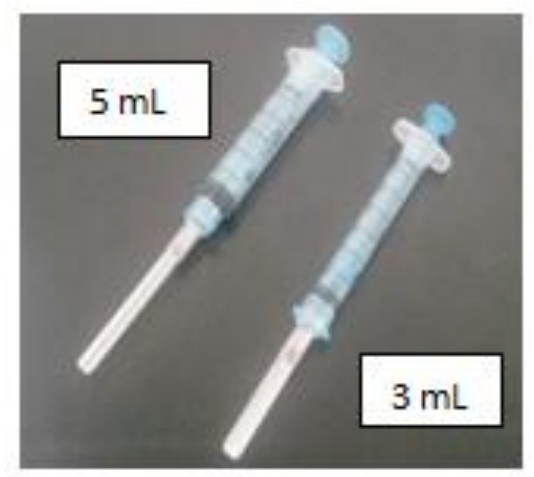

Considerando-se como referência nos dois casos o valor inicial, ou seja, sem adição de soro fisiológico, foram calculados os fatores de correção até se atingir o valor máximo de volume da solução em 3,0 mL. Em todos os casos, os valores de atividade obtidos foram corrigidos para o decaimento da fonte. Desta forma utilizou se a equação:

$$
F_{c v}=\frac{A_{s s}}{A_{c s}}
$$

onde,

$\mathrm{Fcv}=$ Fator de correção para o volume

Ass $=$ Valor de atividade inicial, sem adição de soro fisiológico.

Acs $=$ Valor de atividade após a adição de soro fisiológico

\section{RESULTADOS E DISCUSSÕES}

Nas tabelas 1, 2,3e 4 são apresentados os fatores de correção obtidos utilizando a amostra inicial de $0,5 \mathrm{~mL}$ de ${ }^{99 m} \mathrm{Tc}$, puro e acrescido de diferentes volumes de soro fisiológico, para cada um dos diferentes recipientes utilizados. 
Tabela 1. Fator de correção para o frasco $10 \mathrm{R}$, partindo de uma amostra de $0,5 \mathrm{~mL}$ e acrescentando soro fisiológico até atingir o total de $3 \mathrm{~mL}$.

\begin{tabular}{|c|c|}
\hline \multicolumn{2}{|l|}{$10 \mathrm{R}$} \\
\hline Volume & Fator de correção \\
\hline 0,5 mL ${ }^{99 m}$ Tc (Puro) & 1 \\
\hline $0,5 \mathrm{~mL}^{99 \mathrm{~m}} \mathrm{Tc}+0,5 \mathrm{~mL}$ soro & $1,0004 \pm 0,0005$ \\
\hline $0,5 \mathrm{~mL}^{99 \mathrm{~m}} \mathrm{Tc}+1,0 \mathrm{~mL}$ soro & $0,9995 \pm 0,0004$ \\
\hline $0,5 \mathrm{~mL}^{99 \mathrm{~m}} \mathrm{Tc}+1,5 \mathrm{~mL}$ soro & $1,0024 \pm 0,0004$ \\
\hline $0,5 \mathrm{~mL}^{99 \mathrm{~m}} \mathrm{Tc}+2,0 \mathrm{~mL}$ soro & $1,0034 \pm 0,0005$ \\
\hline $0,5 \mathrm{~mL}^{99 \mathrm{~m}} \mathrm{Tc}+2,5 \mathrm{~mL}$ soro & $1,0034 \pm 0,0004$ \\
\hline
\end{tabular}

Tabela 2. Fatores de correção para o frasco IPEN, partindo de uma amostra de 0,5 mL e acrescentando soro fisiológico até atingir o total de $3 \mathrm{~mL}$.

\begin{tabular}{lc}
\hline \multicolumn{2}{c}{ IPEN } \\
\hline Volume & Fator de correção \\
$0,5 \mathrm{~mL}^{99 \mathrm{~m}} \mathrm{Tc}$ (Puro) & 1 \\
$0,5 \mathrm{~mL}^{99 \mathrm{~m}} \mathrm{Tc}+0,5 \mathrm{~mL}$ soro & $1,0009 \pm 0,0006$ \\
$0,5 \mathrm{~mL}^{99 \mathrm{~m}} \mathrm{Tc}+1,0 \mathrm{~mL}$ soro & $1,0025 \pm 0,0006$ \\
$0,5 \mathrm{~mL}^{99 \mathrm{~m}} \mathrm{Tc}+1,5 \mathrm{~mL}$ soro & $1,0055 \pm 0,0005$ \\
$0,5 \mathrm{~mL}^{99 \mathrm{~m}} \mathrm{Tc}+2,0 \mathrm{~mL}$ soro & $1,0066 \pm 0,0005$ \\
$0,5 \mathrm{~mL}^{99 \mathrm{~m}} \mathrm{Tc}+2,5 \mathrm{~mL}$ soro & $1,0051 \pm 0,0005$ \\
\hline
\end{tabular}

Tabela 3. Fator de correção para a seringa $3 \mathrm{~mL}$, partindo de uma amostra de $0,5 \mathrm{~mL}$ e acrescentando soro fisiológico até atingir o total de $3 \mathrm{~mL}$.

\begin{tabular}{lc}
\hline \multicolumn{2}{c}{ Seringa 3,0 mL } \\
\hline \multicolumn{1}{c}{ Volume } & Fator de correção \\
$0,5 \mathrm{~mL}^{99 \mathrm{~m}} \mathrm{Tc}$ (Puro) & 1 \\
$0,5 \mathrm{~mL}^{99 \mathrm{~m}} \mathrm{Tc}+0,5 \mathrm{~mL}$ soro & $1,0052 \pm 0,0005$ \\
$0,5 \mathrm{~mL}^{99 \mathrm{~m}} \mathrm{Tc}+1,0 \mathrm{~mL}$ soro & $1,0093 \pm 0,0003$ \\
$0,5 \mathrm{~mL}^{99 \mathrm{~m}} \mathrm{Tc}+1,5 \mathrm{~mL}$ soro & $1,0118 \pm 0,0003$ \\
$0,5 \mathrm{~mL}^{99 \mathrm{~m}} \mathrm{Tc}+2,0 \mathrm{~mL}$ soro & $1,0095 \pm 0,0003$ \\
$0,5 \mathrm{~mL}^{99 \mathrm{~m}} \mathrm{Tc}+2,5 \mathrm{~mL}$ soro & $1,0294 \pm 0,0003$ \\
\hline
\end{tabular}


Tabela 4. Fator de correção para a seringa $5 \mathrm{~mL}$, partindo de uma amostra de $0,5 \mathrm{~mL}$ e acrescentando soro fisiológico até atingir o total de $3 \mathrm{~mL}$.

\begin{tabular}{lc}
\hline \multicolumn{2}{c}{ Seringa 5,0 mL } \\
\hline $\begin{array}{c}\text { Volume } \\
0,5 \mathrm{~mL}^{99 \mathrm{~m}} \mathrm{Tc} \text { (Puro) }\end{array}$ & Fator de correção \\
$0,5 \mathrm{~mL}^{99 \mathrm{~m}} \mathrm{Tc}+0,5 \mathrm{~mL}$ soro & $1,0042 \pm 0,0005$ \\
$0,5 \mathrm{~mL}^{99 \mathrm{~m}} \mathrm{Tc}+1,0 \mathrm{~mL}$ soro & $1,0061 \pm 0,0005$ \\
$0,5 \mathrm{~mL}^{99 \mathrm{~m}} \mathrm{Tc}+1,5 \mathrm{~mL}$ soro & $1,0095 \pm 0,0005$ \\
$0,5 \mathrm{~mL}^{99 \mathrm{~m}} \mathrm{Tc}+2,0 \mathrm{~mL}$ soro & $1,0134 \pm 0,0005$ \\
$0,5 \mathrm{~mL}^{99 \mathrm{~m}} \mathrm{Tc}+2,5 \mathrm{~mL}$ soro & $1,0160 \pm 0,0005$ \\
\hline
\end{tabular}

Os resultados obtidos mostram que, para os frascos, a alteração máxima obtida foi menor do que $1 \%$, para o frasco $10 \mathrm{R}$, com um volume total de $1,5 \mathrm{~mL}$ de amostra. Já no caso das seringas a variação máxima encontrada foi de $3 \%$, para a seringa de $3 \mathrm{~mL}$ com volume total de $3 \mathrm{~mL}$ de amostra. Os resultados obtidos para as amostras iniciais de 1,0 mL estão apresentados nas tabelas 5, 6,7 e 8, nas mesmas condições anteriores, ou seja, são apresentados os fatores de correção referentes a 1,0 $\mathrm{mL}$ de ${ }^{99 \mathrm{~m}} \mathrm{Tc}$, puro e diluído em soro.

Tabela 5. Fator de correção para o frasco $10 \mathrm{R}$, partindo de uma amostra de $1,0 \mathrm{~mL}$ e acrescentando soro fisiológico até atingir o total de $3 \mathrm{~mL}$.

\begin{tabular}{ccc}
\hline & $\mathbf{1 0} \mathbf{R}$ \\
\hline & Volume & Fator de correção \\
$1,0 \mathrm{~mL}$ & 1 \\
$1,0 \mathrm{~mL}$ & $\mathrm{Tc}$ ( Puro) & $1,0005 \pm 0,0004$ \\
$1,0 \mathrm{~mL}$ & $\mathrm{Tc}+0,5 \mathrm{~mL}$ soro & $1,0322 \pm 0,0004$ \\
$1,0 \mathrm{~mL}^{99 \mathrm{~m}} \mathrm{Tc}+1,0 \mathrm{~mL}$ soro & $1,5 \mathrm{~mL}$ soro & $1,0365 \pm 0,0004$ \\
$1,0 \mathrm{~mL}^{99 \mathrm{~m}} \mathrm{Tc}+2,0 \mathrm{~mL}$ soro & $1,0393 \pm 0,0004$ \\
\hline
\end{tabular}


Tabela 6. Fator de correção para o frasco IPEN, partindo de uma amostra de 1,0 mL e acrescentando soro fisiológico até atingir o total de $3 \mathrm{~mL}$.

\begin{tabular}{cc}
\hline \multicolumn{2}{c}{ IPEN } \\
\hline Volume & Fator de correção \\
$1,0 \mathrm{~mL}^{99 \mathrm{~m}} \mathrm{Tc}($ Puro $)$ & 1 \\
$1,0 \mathrm{~mL}^{99 \mathrm{~m}} \mathrm{Tc}+0,5 \mathrm{~mL}$ soro & $1,0003 \pm 0,0003$ \\
$1,0 \mathrm{~mL}^{99 \mathrm{~m}} \mathrm{Tc}+1,0 \mathrm{~mL}$ soro & $1,0003 \pm 0,0004$ \\
$1,0 \mathrm{~mL}^{99 \mathrm{~m}} \mathrm{Tc}+1,5 \mathrm{~mL}$ soro & $1,0004 \pm 0,0003$ \\
$1,0 \mathrm{~mL}^{99 \mathrm{~m}} \mathrm{Tc}+2,0 \mathrm{~mL}$ soro & $1,0005 \pm 0,0003$ \\
\hline
\end{tabular}

Tabela 7. Fator de correção para seringa $3 \mathrm{~mL}$, partindo de uma amostra de 1,0 mL e acrescentando soro fisiológico até atingir o total de $3 \mathrm{~mL}$.

\begin{tabular}{|c|c|}
\hline \multicolumn{2}{|c|}{ Seringa $3,0 \mathrm{~mL}$} \\
\hline Volume & Fator de correção \\
\hline $1,0 \mathrm{~mL}^{99 \mathrm{~m}} \mathrm{Tc}$ (Puro) & 1 \\
\hline $1,0 \mathrm{~mL}^{99 \mathrm{~m}} \mathrm{Tc}+0,5 \mathrm{~mL}$ soro & $1,0003 \pm 0,0004$ \\
\hline $1,0 \mathrm{~mL}^{99 \mathrm{~m}} \mathrm{Tc}+1,0 \mathrm{~mL}$ soro & $0,0988 \pm 0,0003$ \\
\hline $1,0 \mathrm{~mL}^{99 \mathrm{~m}} \mathrm{Tc}+1,5 \mathrm{~mL}$ soro & $1,0148 \pm 0,0003$ \\
\hline $1,0 \mathrm{~mL}^{99 \mathrm{~m}} \mathrm{Tc}+2,0 \mathrm{~mL}$ soro & $1,0174 \pm 0,0004$ \\
\hline
\end{tabular}

Tabela 8. Fator de correção para a seringa $5 \mathrm{~mL}$, partindo de uma amostra de $1,0 \mathrm{~mL}$ e acrescentando soro fisiológico até atingir o total de $3 \mathrm{~mL}$.

\begin{tabular}{|c|c|}
\hline \multicolumn{2}{|c|}{ Seringa 5,0 mL } \\
\hline Volume & Fator de correção \\
\hline $1,0 \mathrm{~mL}^{99 \mathrm{~m}} \mathrm{Tc}$ ( Puro) & 1 \\
\hline $1,0 \mathrm{~mL}^{99 \mathrm{~m}} \mathrm{Tc}+0,5 \mathrm{~mL}$ soro & $1,0070 \pm 0,0003$ \\
\hline $1,0 \mathrm{~mL}^{99 \mathrm{~m}} \mathrm{Tc}+1,0 \mathrm{~mL}$ soro & $1,0084 \pm 0,0003$ \\
\hline $1,0 \mathrm{~mL}^{99 \mathrm{~m}} \mathrm{Tc}+1,5 \mathrm{~mL}$ soro & $1,0130 \pm 0,0003$ \\
\hline $1,0 \mathrm{~mL}^{99 \mathrm{~m}} \mathrm{Tc}+2,0 \mathrm{~mL}$ soro & $1,0141 \pm 0,0004$ \\
\hline
\end{tabular}


Neste caso, encontrou-se uma variação máxima de até $4 \%$ para o frasco 10R, com volume total de $3 \mathrm{~mL}$. Já no caso das seringas a variação máxima encontrada foi de 1,7\% para a seringa de $3 \mathrm{~mL}$ para o volume total de $3 \mathrm{~mL}$.

\section{CONCLUSÃO}

No experimento 1 verificou-se uma variação máxima de quase $3 \%$ para a seringa de $3 \mathrm{~mL}$ e 1,6\% para a seringa de $5 \mathrm{~mL}$, quando adicionado soro fisiológico. No experimento 2 observou-se que o frasco 10R teve uma maior variação em relação ao frasco IPEN, sendo está variação máxima de quase $4 \%$. Provavelmente as variações das leituras dos frascos de vidro com a amostra de ${ }^{99 \mathrm{~m}} \mathrm{Tc}$ com a adição de soro fisiológico, ocorreram devido a mudança da geometria da amostra no frasco devido a variação do volume. No caso das seringas essas variações devem-se a geometria e volume da amostra. Desta forma as maiores fontes de erros nas medições realizadas com um ativímetro estão relacionadas com a espessura, o tamanho e o volume do frasco que contém o radiofármaco, considerando que a resposta de um ativímetro depende do frasco a ser utilizado.

Assim sendo é fundamental a aplicação de fatores de correção toda a vez que se altera qualquer um desses fatores, evitando assim subestimar ou superestimar a dose no paciente.

Além disso, é importante lembrar que para obter-se uma medição com mais confiabilidade está na forma com que o trabalhador manipula esses radiofármacos. Um treinamento adequado é essencial para minimizar os erros ocasionados no momento das medições.

\section{AGRADECIMENTOS}

Os autores agradecem o apoio financeiro parcial da Fundação de Amparo à Pesquisa do Estado de São Paulo (FAPESP), Conselho Nacional de Desenvolvimento Científico e Tecnológico (CNPq), Coordenação de Aperfeiçoamento de Pessoal de Nível Superior (CAPES) e Ministério da Ciência e Tecnologia (MCT, Projeto: Instituto Nacional de Ciência e Tecnologia (INCT) EM Metrologia das Radiações na Medicina), Brasil. 


\section{REFERÊNCIAS}

1 .Syringe Calibration Factors and Volume Correction Factors for the NPL Second Standard Radionuclide Calibrator, NPL REPORT CIRM 56, (2002).

2 .MARTINS E.W.,POTIENS, M. P. A. Determination of the influence factors of the radiopharmaceutical vials dimensions used for activimeter calibration at IPEN. Applied Radiation and Isotopes, v.70, p. 1281-1283, 2012.

3. KUAHARA, L.T., CORRÊA, E. L., POTIENS M. P. A., Influence of the radioactive source position inside the well-type ionization chamber.8th Brazilian Congress on Metrology, $29 \mathrm{de}$ Novembro a 04 Dezembro 2015, Bento Gonçalves-RS.

4 .INTERNATIONAL ATOMIC ENERGY AGENCY. Quality assurance for radioactivity measurement in Nuclear Medicine.Technical Report Series. IAEA, TRS 454, Vienna, 2006

5 .EUROPEAN PHARMACOPOEIA: European Directorate for the quality of Medicines (EDQM), $4^{\text {th }}$ ed. Council of Europe, Strasbourg, 2002.

6 . COMISSÃO NACIONAL DE ENERGIA NUCLEAR, CNEN-NN-3.05, Requisitos de radioproteção e segurança para serviços de medicina nuclear. Resolução CNEN- 156/13. Diário Oficial da União, 2013.

7 .CORREAA, E.L., KUAHARA, L.T., POTIENS, M. P. A. Quality control tests of an activity meter to be used as reference for an in situ calibration methodology. 24 a 29 de Novembro 2013. International Nuclear Atlantic Conference - INAC 2013.

8 .KUAHARA, L. T., MARTINS, E. W., DIAS C. R., CORREAA, E. L., POTIENS, M. P. A., JUNIOR, A. C. R. Testes de controle de qualidade em calibradores de dose utilizados em laboratórios de pesquisa do IPEN. 24 a 29 de Novembro 2013. International Nuclear Atlantic Conference - INAC 2013. 